\title{
Lepton Asymmetry and Neutrino Oscillations Interplay
}

\author{
Daniela Kirilova $^{\dagger}$ \\ ${ }^{\dagger}$ Institute of Astronomy and NAO, Bulgarian Academy of Sciences, Sofia
}

\begin{abstract}
We discuss the interplay between lepton asymmetry $\mathrm{L}$ and $\nu$ oscillations in the early Universe. Neutrino oscillations may suppress or enhance previously existing L. On the other hand L is capable to suppress or enhance neutrino oscillations. The mechanism of L enhancement in MSW resonant $\nu$ oscillations in the early Universe is numerically analyzed. L cosmological effects through $\nu$ oscillations are discussed. We discuss how L may change the cosmological BBN constraints on neutrino and show that BBN model with $\nu_{e} \leftrightarrow \nu_{s}$ oscillations is extremely sensitive to $\mathrm{L}$ - it allows to obtain the most stringent constraints on $\mathrm{L}$ value. We discuss also the cosmological role of active-sterile $\nu$ mixing and $\mathrm{L}$ in connection with the indications about additional relativistic density in the early Universe, pointed out by BBN, CMB and LSS data and the analysis of global $\nu$ data.
\end{abstract}

keywords: lepton asymmetry, neutrino oscillations, BBN, excess radiation

\section{Introduction}

Lepton asymmetry $(\mathrm{L})$ of the Universe is not measured yet and may be orders of magnitude bigger than the baryon asymmetry $\beta \sim 6.10^{-10}$, which was measured with great precision from Cosmic Microwave Background (CMB) and Big Bang Nucleosynthesis (BBN) data. L is usually defined as $L=\left(N_{l}-N_{\bar{l}}\right) / N_{\gamma}$, where $N_{l}$ is the number density of leptons, $N_{\bar{l}}$ of antileptons, while $N_{\gamma}$ is the number density of photons. Considerable L might be contained only in $\nu$ sector. Thus, the detection of the Cosmic Neutrino Background would provide $\mathrm{L}$ direct measurement. Till then $\mathrm{L}$ is measured indirectly by its influence on observable relics of the Universe. The abundances of the primordially produced light elements during BBN provide such a sensitive test of $\mathrm{L}$. 
Main cosmological effects of $\mathrm{L}$ on BBN are its dynamical and kinetic effect. The dynamical effect of $L$ consists in the increase of the radiation energy density of the Universe due to non-zero L: $\delta \rho_{r}=\left[1+7 / 8(4 / 11)^{4 / 3} \delta N_{e f f}\right] \rho_{\gamma}$, where $\Delta N_{\text {eff }}=15 / 7\left[(\xi / \pi)^{4}+2(\xi / \pi)^{2}\right]$, where $\xi=\mu / T$ is the $\nu$ degeneracy parameter. This effect leads to faster Universe expansion $H=(8 / 3 \pi G \rho)^{1 / 2}$, which leads to earlier freezing of nucleons and hence influences BBN produced elements. The kinetic effect is noticeable for big enough $|L|>0.01$ in the $\nu_{e}$ sector and is due to different number densities of $\nu_{e}$ and $\overline{\nu_{e}}$, which participates in neutron-proton transfers: $\nu_{e}+n \leftrightarrow p+e^{-}, \overline{\nu_{e}}+p \leftrightarrow n+e^{+}$in the pre-BBN epoch and, correspondingly, BBN yields (see ref. [1] and refs there in).

Besides these well known effects L may influence BBN through its interplay with neutrino oscillations. Unlike previous effects, this L effect on BBN, called further indirect kinetic effect, may be noticeable for much smaller L values, $L<<0.01$ [2]. Hence BBN with oscillations provides the possibility to measure and/or constrain tiny $\mathrm{L}$ with values close to $\beta$.

In the next section the interplay between $\mathrm{L}$ and $\nu$ oscillations is described. In the third section the indirect kinetic effect of $\mathrm{L}$ on $\mathrm{BBN}$ is studied and the possibility to constrain $\mathrm{L}$ via $\mathrm{BBN}$ with $\nu$ oscillations is discussed. L role as a solution of the problem of the excess radiation density in the Universe is provided in the fourth section.

\section{Interplay between $\mathrm{L}$ and neutrino oscillations in the early Universe}

Small $L<<0.01$ has negligible dynamic and direct kinetic effect. Nevertheless, due to its interplay with $\nu$ oscillations such $\mathrm{L}$ is capable of changing $\nu$ number densities, $\nu$ distribution and spectrum distortion and changing $\nu$ oscillations pattern (suppressing or enhancing oscillations), by which it influences nucleons kinetics and finally BBN production of light elements [2]. This effect was proven to persist down to $L \sim 10^{-8}[2,3,4]$.

We studied numerically the interplay between tiny L, $10^{-10}<L<10^{-4}$, and electronsterile $\nu_{e} \leftrightarrow \nu_{s}$ oscillations, effective after active $\nu$ decoupling $\delta m^{2} \sin ^{4} 2 \theta \leq 10^{-7} \mathrm{eV}^{2}$.

It is known that active-sterile oscillations may change neutrino-antineutrino asymmetry of the medium, suppress or enhance it $[2,3,4,5,6,8,9,10,11,12,13,14,15,16]$. 1 On the other hand L influences $\nu$ propagation. Qualitatively this influence may be described as follows: The average potentials $V_{f}$ for $\nu$ depend on the particle asymmetries

\footnotetext{
${ }^{1}$ There are other cosmological effects of active-sterile neutrino oscillations, like excitation of additional light particles into equilibrium $[5,6]$ and distortion $\nu$ energy distribution $[7,8]$.
} 
of different constituents of the medium and they differ for different neutrino types due to different interactions with the particles of the plasma [17]:

$$
V_{f}=\sqrt{2} G_{F} Q N_{\gamma} / M_{W}^{2} \pm L N_{\gamma}
$$

where $f=e, \mu, \tau$, "minus" corresponds to $\nu$ and "plus" to $\bar{\nu}, Q \sim-E T, L \sim-L_{\alpha}$, $L^{\alpha}$ is given through the fermion asymmetries of the plasma (in the discussed case $L \sim$ $\left.2 L_{\nu_{e}}+L_{\nu_{\mu}}+L_{\nu_{\tau}}\right)$.

In the adiabatic case the effect of the medium can be hidden in the oscillation parameters $\delta m^{2}$ and $\vartheta$ by introducing matter oscillation parameters:

$$
\sin ^{2} \vartheta_{m}=\sin ^{2} \vartheta /\left[\sin ^{2} \vartheta+2 G_{F}\left(\left(Q / M_{W}^{2} \mp L\right) N_{\gamma} / \delta m^{2}-\cos 2 \vartheta\right)^{2}\right]
$$

L, as a characteristic of the medium, may suppress oscillations by decreasing their amplitude, or enhance oscillation transfer in case resonant condition between the parameters of the medium and the oscillation parameters holds:

$$
2 G_{F}\left(Q / M_{W}^{2} \mp L\right) N_{\gamma}=\cos 2 \vartheta \times \delta m^{2}
$$

In the early Universe at high temperature $Q / M_{W}^{2}>L$ resonant oscillations both for $\nu$ and $\bar{\nu}$ are possible if $\delta m^{2}<0$. With the cooling of the Universe when L begins to dominate, $Q / M_{W}^{2}<L$, resonant transfer for antineutrinos in case $\delta m^{2}<0$, or for neutrinos if $\delta m^{2}>0$ is possible. ${ }^{2}$ Thus neutrino propagation and resonance in the neutrino sector differs from that of antineutrino for non-zero L. Due to L influence of the $\nu$ propagation L may change $n_{\nu}$, its spectrum distribution and oscillation pattern.

This simplified description of the medium influence is applicable in the equilibrium situation, when working in terms of average $\nu$ momentum and particle densities is reasonable. In the nonequilibrium situation, when spectrum distribution of $\nu$ is considerable, as is the case of late electron-sterile oscillations discussed here, it strongly effects both $\nu$ propagation and L evolution. Hence, for the correct description of the neutrino - asymmetry interplay it is essential to provide an accurate account of the neutrino spectrum distortion due to $\mathrm{L}$ and oscillations. 3

\footnotetext{
${ }^{2}$ The resonant condition (3) for $Q=0$ was first studied and is known as Mikheev-Smirnov-Wolfenstein effect [18].

${ }^{3}$ For example, in the nonequilibrium situation, when spectrum distribution of $\nu$ was properly described, simultaneous resonance transfer was found possible also in the $\delta m^{2}<0$ case due to the "resonant wave" passing through the neutrino distribution [2].
} 


\subsection{Exact description of the propagation of neutrinos and $\mathrm{L}$ evolution}

The equations governing $\nu$ evolution, in terms of neutrino density matrix in momentum space, are given below. They account simultaneously for neutrino-L interplay, Universe expansion, $\nu$ oscillations and $\nu$ forward scattering and describe precisely $\nu$ energy distribution [8]. Our numerical analysis of $\mathrm{L}$ and $\nu$ propagation was based on these equations:

$$
\begin{gathered}
\partial \rho(t) / \partial t=H p_{\nu}\left(\partial \rho(t) / \partial p_{\nu}\right)+ \\
+i\left[\mathcal{H}_{o}, \rho(t)\right]+i \sqrt{2} G_{F}\left(\mathcal{L}-Q / M_{W}^{2}\right) N_{\gamma}[\alpha, \rho(t)]+\mathrm{O}\left(G_{F}^{2}\right) \\
\partial \bar{\rho}(t) / \partial t=H p_{\nu}\left(\partial \bar{\rho}(t) / \partial p_{\nu}\right)+ \\
+i\left[\mathcal{H}_{o}, \bar{\rho}(t)\right]+i \sqrt{2} G_{F}\left(-\mathcal{L}-Q / M_{W}^{2}\right) N_{\gamma}[\alpha, \bar{\rho}(t)]+\mathrm{O}\left(G_{F}^{2}\right) . \\
L_{\nu_{e}} \sim \int \mathrm{d}^{3} p\left(\rho_{L L}-\bar{\rho}_{L L}\right) / N_{\gamma}
\end{gathered}
$$

where $\alpha_{i j}=U_{i e}^{*} U_{j e}, \nu_{i}=U_{i l} \nu_{l}(l=e, s) . \mathcal{H}_{o}$ is the free $\nu$ Hamiltonian. $Q$ arises as an $W / Z$ propagator effect, $Q \sim E_{\nu} T$. $\mathcal{L} \sim 2 L_{\nu_{e}}+L_{\nu_{\mu}}+L_{\nu_{\tau}}, L_{\mu, \tau} \sim\left(N_{\mu, \tau}-N_{\bar{\mu}, \bar{\tau}}\right) / N_{\gamma}$. At decoupling of $\nu_{e} \nu_{s}$ was assumed empty. ${ }^{4}$

Due to $L$ the equations are coupled integro-differential. $L$ leads to different evolution of $\nu$ from $\bar{\nu}$ due to the different sign of $\mathrm{L}$ in the equations. Numerical analysis of the evolution of $\nu$ ensembles, evolution of $L$, and also the evolution of nucleons for the entire range of oscillation parameters and for the temperature range [2 MeV, $0.3 \mathrm{MeV}]$ and for $10^{-10}<L<0.01$ was provided in non-resonant $\nu$ oscillations case. In case of resonant oscillations $\mathrm{L}$ initial value was taken to be $L_{i} \sim \beta$. We have described precisely $\nu$ momenta distribution: 5000 bins were used in the non-resonant oscillations case, and up to 10000 in the resonant case.

In case of nonresonant oscillations and relic $L$ the following relations describe with good accuracy the exact behavior of $L$ and $L$-oscillations interplay: $L \geq 10^{-7}$ enhances oscillations, $L>0.1\left(\delta \mathrm{m}^{2} / \mathrm{eV}^{2}\right)^{2 / 3}$ suppresses oscillations, and asymmetries $L>$ $\left(\delta m^{2} / \mathrm{eV}^{2}\right)^{2 / 3}$ inhibit oscillations. For illustration of the exact dependence see the figures in ref. [4].

In case of resonant $\nu_{e} \leftrightarrow \nu_{s}$ oscillations the evolution of $L$ has a rapid oscillatory behavior. The region of parameter space for which a generation of $\mathrm{L}$ is possible was found

\footnotetext{
${ }^{4}$ The case of non-zero population of $\nu_{s}$ was considered in refs. [15, 19, 20].
} 
$\left|\delta m^{2}\right| \sin ^{4} 2 \theta \leq 10^{-9.5} \mathrm{eV}^{2} .{ }^{5}$ A maximum possible growth of $L$ by 5 orders of magnitude was determined.

$\mathrm{L}$ role in BBN with $\nu$ oscillations was numerically studied as well. The change in $\mathrm{BBN}$ constraints on oscillation parameters due to $\mathrm{L}$ and $\mathrm{BBN}$ constraints on $\mathrm{L}$ in case of $\nu_{e} \leftrightarrow \nu_{s}$ are presented in the next section.

\section{BBN with active-sterile neutrino oscillations and lepton asymmetry}

Big Bang Nucleosynthesis is theoretically well established due to the precise data on nuclear processes rates, known from laboratory experiments at the energies relevant to BBN epoch, and the precise data on the light elements abundances D, He and Li. Besides, the baryon-to-photon ratio, which is the only parameter in the standard BBN, has been independently measured with good accuracy by CMB precision data. Therefore, BBN is used as the most early and precision probe for physical conditions in early Universe and, hence, presents the best astrophysical and cosmological probe for new physics and microphysics, relevant at BBN energies.

Being the best speedometer at radiation dominated stage BBN was used to probe $\nu$ properties, the number of light species, $\nu$ oscillations, distortion in $\nu$ distribution, etc. Due to its sensitivity to the expansion rate and to the nucleons kinetics in the pre-BBN epoch it has been shown that BBN presents also the most exact leptometer (see ref. [4] and references there in). Thus BBN studies allow to put stringent limits on oscillation parameters $[5,7,6,8,21,12,13,15,19,20,23]$ and on $\mathrm{L}$ in the presence of electron-sterile oscillations $[2,3,4]$.

\section{$2.1 \quad$ BBN constraints on $\mathrm{L}$}

Eventual $\nu$ degeneracies of flavor neutrinos will equilibrate before BBN due to flavor $\nu$ oscillations, having in mind recently measured value $\theta_{13}$. Then BBN constraints on $\mathrm{L}$ read $[24,25,26,27,28,29]$ :

$$
|L|<0.1
$$

These constraints allow the possibility of L orders of magnitude larger than the measured $\beta$. However, for such small $L$ the dynamical cosmological effect of $L$ isnegligible.

\footnotetext{
${ }^{5}$ The instability region is slightly more stringent than the existing in literature for other oscillation models ref. [22].
} 
Hence, that small $\mathrm{L}$ in the flavor $\nu$ sector cannot mimic the extra relativistic degrees of freedom during $\mathrm{BBN}$, which seem to be required by recent analysis of cosmological and neutrino oscillations data (to be discussed in more detail in the fourth section).

\section{$2.2 \quad \mathrm{~L}$ and $\mathrm{BBN}$ with late electron sterile $\nu$ oscillations}

In case of $L \sim \beta$ stringent $\mathrm{BBN}$ constraints on active-sterile oscillation parameters exist. The presence of $L>\beta$ may change considerably BBN constraints on oscillations parameters due to $\mathrm{L}$ indirect kinetic effect.

In $\mathrm{BBN}$ with electron-sterile $\nu$ oscillations $Y_{p}$ decreases at small mixing parameters values due to $\mathrm{L}$ growth caused by resonant $\nu$ transfer, thus at these mixing angles oscillations generated $L$ relaxes the $B B N$ constraints on oscillations $[8,12,4]$.

In case of relic $L>\beta$ the presence of such $L$ relaxes the BBN bounds on neutrino oscillation parameters in case of electron-sterile non-resonant neutrino oscillations at maximal mixing and strengthens them at small mixing angles. In this case depending on the asymmetry-oscillations interplay the asymmetry may enhance, suppress or stop $\nu$ oscillations, reflecting correspondingly to strengthening, relaxation or elimination of the BBN constraints. In the last case the approximate BBN constraint reads:

$$
\delta m^{2}\left(e V^{2}\right)<L^{3 / 2}
$$

Vice versa, this constraint can be considered also as a cosmological constraint on L from BBN with electron-sterile neutrino oscillations. Thus, L generated in the electron-sterile sector may (partially) suppress the oscillations in other sectors, hindering equalization of chemical potentials, thus relaxing the stringent BBN bound. The presence of $\mathrm{L}$ (no matter how generated), capable to suppress oscillations, may lead to only partial population of the sterile neutrino. Vise versa L values capable to enhance oscillations will bring faster the sterile state into equilibrium.

The model of BBN with $\nu_{e} \leftrightarrow \nu_{s}$, effective after electron $\nu$ decoupling presents the possibility to feel extremely small asymmetries $L>10^{-8}$ due to the indirect kinetic effect of L (see ref. [4]). This sensitivity allows to derive stronger constraints on L. Namely, equation (5) can be used to put the following BBN constraint on L: Having the indications for active-sterile oscillations with $\delta m^{2} \sim 10^{-5}$ [30] and replacing this value in eq.(5), a much stronger upper limit on $\mathrm{L}$ (than the one presented in eq.(4)) follows $L<10^{-3.3}$. 


\section{$3 \quad \mathrm{~L}$ and excess radiation density in the Universe}

In recent years an increasing number of cosmological indications suggesting excess relativistic density, corresponding to different epochs, appeared [31, 32, 33, 34, 35, 36, 37]. 6 Besides, $\nu$ oscillations data require 1 or 2 additional sub-eV sterile neutrino, participating into oscillations with flavor neutrinos with higher mass differences values, than the ones required by solar and atmospheric $\nu$ oscillations experiments $[40,42,43,44,45]$. It is interesting if cosmology allows 2 light additional sterile neutrinos and if they can explain the excess relativistic density.

Additional light sterile neutrinos with the mixing and mass differences estimated by $\nu$ oscillations data with mass differences in the eV range will be brought into equilibrium in the early Universe. BBN favors the presence of one such $\nu_{s}$ but He and D data excludes 2 fully thermalized $\nu_{s}$. Besides, neutrinos in sub-eV range produce too much hot dark matter $[46,47]$. Thus, two additional $\nu_{s}$ are in tension both with BBN and with LSS requirements $[48,49,36]$.

L, namely its dynamical and direct kinetic effects, has been considered as an explanation of the excess radiation. It was shown that excess radiation cannot be explained by degenerate BBN [29]. However, the presence of $\mathrm{L}$ may be the solution in case its value is enough to suppress active-sterile oscillations so that $\nu_{s}$ are not fully thermalized $[6,9,2,41]$. Our estimation of the value of $L$ necessary to suppress oscillations and achieve the suppression of $\nu_{s}$ production is $L \geq 0.08$. This is higher than the values discussed by refs.[41, 51], that found $|L|>10^{-2}$. The difference might be due to different approximations used or to the fact that previous studies do not account precisely for the $\nu$ energy distribution.

Thus, in modified BBN with $\nu$ oscillations and high enough $\mathrm{L}$ the models with additional light sterile neutrinos may be allowed. To obtain the exact $\mathrm{L}$ value a precise numerical analysis, solving the exact kinetic equations, including all $\nu$ species and accounting for all L effects, discussed above, should be provided.

Hence, the excess relativistic density might point to additional sterile neutrinos and the presence of L. However, there exist other possibilities as well, namely: $\nu$ active-sterile late oscillations leading to the overproduction of He-4 and thus imitating extra radiation, $\mathrm{MeV}$ decaying particles during BBN [53], or other modifications of the standard cosmological model. Future experimental and observational data will choose among different possibilities. In particular, it is expected that Planck data will be able to check with

\footnotetext{
${ }^{6}$ See, however, the recent results [38], which are in agreement with the canonical value of $N_{\text {eff }}$ and also the discussion in ref.[39].
} 
higher sensitivity the status of extra radiation.

\section{Conclusions}

There exists interesting interplay between small L, relic or produced by active-sterile $\nu$ oscillations, and late active-sterile $\nu$ oscillations. A detail numerical analysis of this interplay between lepton asymmetry $L<<0.01$, and $\nu$ oscillations was carried out. The evolution of $\nu$ and $\mathrm{L}$ was studied using exact kinetic equations for $\nu$ density matrix in momentum space and describing the $\nu$ energy distribution with very high accuracy - up to 10000 bins.

A considerable enhancement of L - by 5 orders of magnitude - was found possible in non-equilibrium electron-sterile resonant neutrino oscillations, effective after the decoupling of active neutrino. The region in the oscillation parameter space of considerable L growth was determined.

The parameter ranges for which relic $\mathrm{L}$ is able to enhance, suppress or inhibit nonresonant electron-sterile oscillations was determined.

Cosmological influence of small $\mathrm{L}$, which do not have direct effect on nucleons kinetics during BBN, was discussed. Such small asymmetries are invisible by CMB at present, but may be felt by BBN. It was shown that $\mathrm{L}$ as small as $10^{-8}$ may be felt by $\mathrm{BBN}$ via oscillations. Also BBN constraints on $\nu$ oscillations parameters depend nontrivially on L.

$\mathrm{L}$ generated by oscillations at small mixing angles suppresses $\nu$ oscillations and reduces the overproduction of $Y_{p}$ and relaxes BBN constraints.

Relic L present during BBN, depending on its value, can strengthen, relax or wave out BBN constraints on oscillations. The value of $\mathrm{L}$, capable to hinder $\nu$ oscillations is determined for different sets of oscillations parameters of the model, a good approximation to the exact value is $L>\left(\delta m^{2} /\left(e V^{2}\right)\right)^{2 / 3}$. On the other hand this can be considered as the most stringent cosmological constraint on $\mathrm{L}$ based on BBN with electron-sterile neutrino oscillations.

Due to its interplay with neutrino oscillations L may play important role for resolving the problem of additional radiation density suggested by cosmological observational data and by $\nu$ oscillations data. In principal BBN with $\mathrm{L}$ may allow $3+1$ and $3+2$ oscillations models, in case L value is high enough to suppress active-sterile oscillations, thus providing incomplete thermalization of $\nu_{s}$ and relaxing $\mathrm{BBN}$ constraints on additional light sterile species.

Acknowledgements. I am glad to thank Theoretical and Computational Physics and Astrophysics Foundation, Sofia University, for the financial support of my travel. I 
acknowledge the financial support by the conference for my participation. I thank the unknown referee for the suggestions and criticism that helped to improve the paper.

\section{References}

[1] Simha, G. Steigman, JCAP0808 011 (2008)

[2] D. Kirilova, M. Chizhov, Nucl. Phys. B534 447 (1998); D. Kirilova, M. Chizhov, Verbier 2000, Cosmology and particle physics, 433 (2001), astro-ph/0101083

[3] Kirilova D., Prog. Part. Nucl. Phys.66, 26 (2011).

[4] Kirilova D., JCAP 06, 007 (2012)

[5] Dolgov A. D. Sov. J. Nucl. Phys. B 506, 7 (1981)

[6] Barbieri R, Dolgov A, Phys. Lett. B 237, 440 (1990)

[7] Kirilova D., 1988 JINR E2-88-301

[8] D. Kirilova, M. Chizhov, Neutrino96, 478 (1996) D. Kirilova, M. Chizhov, Phys.Lett.B393, 375 (1997)

[9] R. Foot, R. R. Volkas Phys.Rev.Lett.75 4350 (1995); Phys.Rev.D55 5147 (1997)

[10] R. Foot, R. R. Volkas, Phys.Rev.D56 6653 (1997);Phys.Rev.D59 029901 (1999)

[11] X. Shi, Phys. Rev. D 542753 (1996)

[12] D. Kirilova, M. Chizhov, Nucl. Phys.B591 457 (2000)

[13] Dolgov A., Villante F., Nucl. Phys. B679, 261 (2004).

[14] R. Foot, M. Thomson, R. Volkas, Phys.Rev.D 53 R5349 (1996)

[15] D. Kirilova, Int. J. Mod. Phys. D13 831 (2004)

[16] P. di Bari, Phys.Rev. D 67, 127301 (2003)

[17] D. Notzold, G. Raffelt, Nucl. Phys. B307, 924 (1988)

[18] S. P. Mikheyev and A. Yu. Smirnov, Sov. J. Nucl. Phys. 42, 913 (1985); Nuovo Cimento9C, 17 (1986); L. Wolfenstein, Phys. Rev. D17, 2369 (1978). 
[19] D. Kirilova, Int. J. Mod. Phys. D,16, 1197 (2007)

[20] D. Kirilova, M. Panayotova, JCAP12, 014 (2006)

[21] Kirilova D, Chizhov M., Phys. Rev.D 58, 073004 (1998)

[22] A. Dolgov, S. Hansen, S. Pastor, D.Semikoz, Astropart.Phys.14 79 (2000)

[23] M. Panayotova Bulg. J. Phys. 38, 341 (2011)

[24] A. Dolgov, S. Hansen, S. Pastor, S.Petcov, G.Raffelt, D.Semikoz, Nucl. Phys. B632, 363 (2002) Y.Y.Y. Wong, Phys. Rev. D66, 025015 (2002);

[25] K.N.Abazajian, J.F. Beacom, N.F. Bell,Phys. Rev. D 66, 013008 (2002)

[26] F.Iocco et al.,Phys.Rep.472, 12009

[27] P. Serpico, G. Raffelt, Phys.Rev.D71 127301 (2005)

[28] S. Pastor, T. Pinto, G. Raffelt, Phys.Rev.Lett.102, 241302 (2009).

[29] G. Mangano, G. Miele, S. Pastor, O. Pisanti, S. Sarikas, Phys.Lett. B708 1 (2012), arXiv:1110.4335; G. Mangano, G. Miele, S. Pastor, O. Pisanti, S. Sarikas, JCAP11, 035 (2011).

[30] P. Holanda, A. Smirnov Phys.Rev.D 83113011 (2011)

[31] Y. Izotov, T. Thuan, Astrophys. J. 710, L67 (2010)

[32] Aver E., Olive K., Skillman E., JCAP 05, 003 (2010)

[33] E. Komatsu et al. (WMAP),Astrophys. J. Suppl.180, 330 (2009) Astrophys.,J. Suppl.192 18 (2011)

[34] R. Keisler et al., Ap.J 743, 28 (2011); arXiv:1105.3182

[35] Dunkley J. et al., A.J 739, 52 (2011); arXiv:1009.0866

[36] Hou Z. et al., arXiv:1104.2333

[37] Riemer-Sorensen S., Parkinson D., Davis T., arXiv:1301.7102

[38] Bennett C. et al., arXiv:1212.5225

[39] Joudaki Sh, Abazajian K., Kaplinghat M., arXiv:1208.4354 
[40] Kopp J., Maltoni, T. Schwetz, Phys. Rev. Lett.107 091801 (2011), arXiv:1103.4570

[41] S. Hannestad, I Tamborra, T. Tram, JCAP1207 025 (2012), arXiv:1204.5861

[42] C. Giunti, M. Laveder, Phys.Rev.D84 093006 (2011), Phys.Rev.D82 093016 (2010), Phys.Lett. B706 200 (2011)

[43] Akhmedov E., T. Schwetz, JHEP 10, 115 (2010) arXiv:1007.4171

[44] Razzaque S., Smirnov A., JHEP 07, 084 (2011) arXiv:1104.1390

[45] Mention G. et al., Phys. Rev. D 83, 073006 (2011); arXiv:1101.2755; Huber P., arXiv:1106.0687

[46] Hamann J. et al. Phys. Rev. Lett. 105, 181301 (2010) arXiv:1006.5276

[47] Dodelson S., Melchiorri A., Slosar A., Phys. Rev. Lett. 97, 14130 (2006); astro/ph 0511500

[48] Giusarma E. et al, Phys. Rev. D 83115023 (2011); arXiv:1102.4774

[49] Nollett K., Holder G., 1112.2683

[50] Hamman J. et al., JCAP 1109, 034 (2011); arXiv:1108.4136

[51] Mirizzi A. et al., Phys. Rev. D 86053009 (2012), arXiv:1206.1046

[52] P. Di Bari, R. Foot, Phys.Rev. D61 105012 (2000)

[53] Kirilova D., School and Workshop on Space Plasma Physics, 83-89, AIP Conf. Proc., 1121, 31.08-07.09, Sozopol, Bulgaria, ed. I. Zhelyazkov, 2009; Dolgov A.,Kirilova D.,Int.J.Mod.Phys. A3, 267 (1988); O. Ruchayskiy, A. Ivashko, JCAP1210 014 (2012), arXiv:1202.2841; J. Menestrina, R. Scherrer, Phys.Rev. D85, 047301 (2012). 